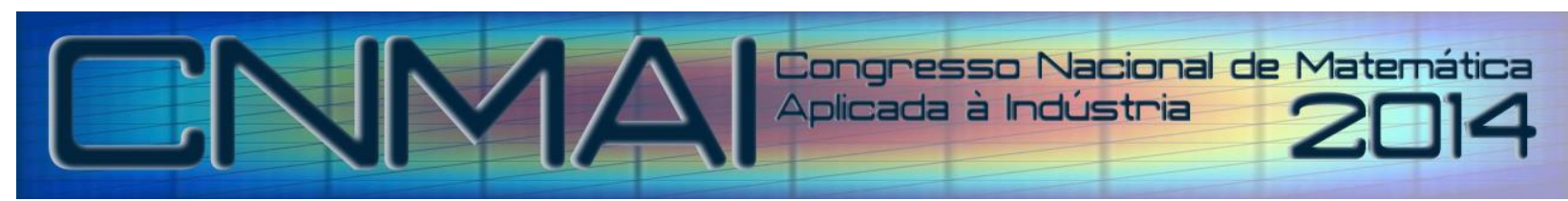

18 a 21 de novembro de 2014, Caldas Novas - Goiás

\title{
PROJETO ÓTIMO DE CIRCUITOS SHUNT PARA O CONTROLE DE VIBRAÇÕES DE ESTRUTURAS COMPOSTAS
}

\author{
Lorrane Pereira Ribeiro, lorranemecatronica@gmail.com ${ }^{1}$ \\ Antônio Marcos Gonçalves de Lima, amglima@mecanica.ufu.br² \\ ${ }^{1,2}$ Universidade Federal de Uberlândia - Faculdade de Engenharia Mecânica - Av. João Naves de Ávila, 2121, Santa \\ Mônica, 38408-100, Uberlândia, Minas Gerais.
}

\begin{abstract}
Resumo: Este trabalho é dedicado à modelagem numérico-computacional de sistemas estruturais do tipo placas planas compostas contendo materiais piezelétricos acoplados a circuitos elétricos passivos (circuitos shunt) monomodais para o controle passivo de vibrações. Serão também empregadas técnicas de otimização multiobjetivo determinística com vistas ao projeto ótimo de tais circuitos elétricos. Para a modelagem por elementos finitos da estrutura composta contendo elementos piezelétricos, ênfase é dada à combinação das teorias da camada equivalente única, em específico, a teoria cisalhante de primeira ordem (First-Order Shear Deformation Theory-FSDT) com a teoria das camadas equivalentes discretas, também conhecida como Layerwise Theory, para a consideração dos campos elétricos que são assumidos discretos ao longo da espessura da placa composta. Todo o procedimento de modelagem foi implementado em ambiente de programação MATLAB®. Neste sentido, está disponível hoje um conjunto de programas de fácil acesso e utilização que permitem o projeto ótimo de circuitos elétricos shunt para serem aplicados a estruturas compostas mais complexas de interesse industrial com vistas ao controle passivo de vibrações de tais sistemas. Os resultados numéricos obtidos em termos das respostas dinâmicas de uma viga composta contendo elemento piezelétrico acoplado a circuitos shunt nominal e otimizados demonstram a eficiência dos mesmos para o controle passivo das vibrações indesejáveis.
\end{abstract}

Palavras-chave: estruturas compostas, materiais piezelétricos, circuitos shunt, controle passivo de vibrações.

\section{INTRODUÇÃO}

No contexto atual de engenharia se observa o crescente aumento do uso de materiais compósitos, motivando inúmeros pesquisadores de todo o mundo quanto à proposição de modelos matemáticos confiáveis e técnicas de simulações numéricas do comportamento dinâmico destes materiais. Desta forma, é oportuno salientar o fato de que nas inúmeras aplicações de materiais compostos, as estruturas estão frequentemente expostas a perturbações estáticas e/ou dinâmicas que afetam significativamente a eficiência desses materiais, levando a um comprometimento da integridade estrutural, podendo levá-las ao colapso. Sendo assim, um aspecto importante a ser investigado, e que tem mobilizado um grande número de pesquisadores em todo o mundo, é o procedimento de modelagem numérico-computacional de estruturas compostas combinadas com técnicas de controle de vibração e ruído (Berthelot, 1992; Faria et al., 2008).

A utilização de materiais compósitos em detrimento aos convencionais é uma realidade, sendo que, tal fato ocorre em virtude das propriedades superiores das estruturas compostas. Pode-se citar como exemplo a relação resistência/peso muito superior desses materiais em relação aos materiais metálicos tradicionais como aço e alumínio (Callister Jr., 2002; Faria, 2006). Outro aspecto importante é que os mesmos podem ser confeccionados de acordo com as necessidades específicas de projeto, apresentando-se como sendo a solução mais adequada quando se necessita de estruturas mais leves e ao mesmo tempo resistentes, ou também outras combinações de propriedades mecânicas difíceis de serem obtidas com os materiais convencionais (Pinheiro et al., 2006).

Em se tratando da modelagem de estruturas compostas contendo sensores e atuadores uma grande diversidade de teorias pode ser encontrada, as quais são utilizadas com base na técnica de elementos finitos, sendo que, cada uma delas apresentam características favoráveis e desfavoráveis, notadamente no que diz respeito à precisão, domínio de aplicação, e esforço computacional envolvido na sua implementação (Faria, 2006; Reddy, 1997). Assim, neste trabalho, o conceito de Camada Equivalente Única é usado para aproximação dos campos de deslocamentos mecânicos e o 
conceito de Camadas Equivalentes Discretas é usado para aproximar os potenciais elétricos. Dentre as várias teorias da primeira categoria, utilizou-se a Teoria da Deformação Cisalhante de Primeira Ordem (First-order Shear Deformation Theory - FSDT), a qual requer funções pertencentes ao espaço $\mathrm{C}^{0}$, ou seja, funções contínuas e pode ser usada para a modelagem de placas e cascas finas ou moderadamente espessas (Cen et al., 2002). Além disso, a FSDT é considerada a teoria que apresenta a melhor relação entre capacidade de predição e custo computacional para uma larga classe de aplicações (Chugal e Shimpi, 2002; Mendonça, 2005).

No que diz respeito ao amortecimento ou controle de vibrações, os materiais piezelétricos vêm sendo utilizados tanto em técnicas ativas como passivas. As técnicas ativas são caracterizadas pela presença dos atuadores, fontes de potência e sistemas de controle. Já nas técnicas passivas as fontes de potência e o sistema de controle são suprimidos, explorando características físicas dos materiais inteligentes, de forma que uma parcela da energia de deformação é absorvida nos modos de interesse e dissipada através de algum mecanismo de dissipação (Viana, 2005).

Clark et al. (1998) definem que piezeletricidade é a propriedade que certos materiais apresentam de se deformarem mecanicamente na presença de um campo elétrico, e no caso inverso, de produzirem uma carga elétrica quando deformados mecanicamente. Esta característica permite que parte da energia mecânica associada à vibração possa ser convertida em energia elétrica e dissipada convenientemente, em um circuito shunt, formando assim o mecanismo de amortecimento passivo.

Dentre os circuitos shunt, o circuito ressonante se destaca. Formado por um indutor e um resistor, este circuito permite a sintonia para qualquer frequência que se queira amortecer, seja uma frequência natural ou não; além disso, algumas melhoras na topologia do circuito possibilitam o amortecimento simultâneo de mais de um modo de vibrar. Do ponto de vista mecânico, o circuito é visto pelo sistema como um absorvedor dinâmico de vibrações (Viana, 2005). Observa-se que pelo fato das frequências envolvidas em problemas de controle passivo de vibrações serem baixas, as indutâncias requeridas no caso de controladores passivos são bastante elevadas, como será mostrado na seção de simulações numéricas. Tal fato leva a necessidade de utilização de indutâncias sintéticas.

Assim, de acordo com o que foi exposto, o principal objetivo deste trabalho é a modelagem numéricocomputacional de estruturas compostas utilizando-se da teoria FSDT, a qual se encontra na categoria das teorias da camada equivalente única, a qual é posteriormente combinada com a teoria discreta, na introdução do elemento piezelétrico na modelagem e, a partir da modelagem da estrutura, realizar o controle passivo de vibrações com a utilização de circuitos shunt.

\section{MODELAGEM MECÂNICA}

As teorias de placas laminadas são classificadas de acordo com as hipóteses cinemáticas adotadas na aproximação das quantidades mecânicas (deslocamentos e deformação). Dentre essas teorias há a seguinte divisão: (a) Teorias baseadas em Camada Equivalente Única (Equivalent Layer Theory): Engloba a Teoria Clássica dos laminados (CLT), a FSDT e a HSDT (High-order Shear Deformation Theory); (b) Teorias baseadas em Camadas Equivalentes Discretas (Discrete Layer Theory): Engloba a Teoria das Camadas independentes, conhecida como Teoria Zig-Zag e também a Teoria das Camadas Dependentes.

Desta forma, a Teoria da Deformação Cisalhante de Primeira Ordem, FSDT, é adotada para a modelagem mecânica. Segundo essa teoria uma linha reta e normal ao plano médio antes da deformação permanece reta, mas, não necessariamente normal a este plano após a deformação. Além disso, ela assume que a deformação cisalhante transversal varia linearmente ao longo da espessura do composto.

Os campos de deslocamentos para a teoria FSDT podem ser assim representados:

$$
\{U(x, y, z, t)\}=[A u(z)]\{\hat{\mathrm{u}}\}
$$

onde $U$ é o vetor de campos de deslocamentos mecânicos, $A u(z)$ é a matriz que contém a coordenada $z$ parametrizada e û representa o vetor dos graus de liberdade elementares mecânicos.

Assumindo-se pequenos deslocamentos e considerando-se os campos de deslocamentos mecânicos previamente definidos, pode-se obter a seguinte expressão entre as deformações e os deslocamentos:

$$
\{\varepsilon\}=[D]\{\hat{\mathrm{u}}\}
$$

onde a matriz $D$ é a matriz de operadores diferenciais.

Foi adotado um sistema de coordenadas global, tendo como coordenadas ( $\mathrm{x}, \mathrm{y}$ e z) comum a toda estrutura. Observa-se nas equações que seguem a utilização de um sistema de coordenadas local $(\xi, \eta, z)$ devido ao fato de neste primeiro momento desejar-se encontrar as matrizes de massa e rigidez elementares, sendo que, a integração para obtenção das mesmas é realizada neste sistema e é utilizado o jacobiano $J$ para a transformação linear de coordenadas.

Devido à natureza ortotrópica dos materiais compostos, suas propriedades mecânicas dependem fortemente das orientações das fibras no estratificado. As matrizes de propriedades mecânicas dos materiais podem ser transformadas por rotação de um ângulo $\theta$ em torno do eixo $z$ usando uma matriz de transformação $T$ (para as propriedades mecânicas) ou $Q$ (para propriedades elétricas), as quais são encontradas na dissertação de Faria (2006). A rotação para o caso das propriedades mecânicas é $\left[C_{t}\right]=[T][C][T]^{T}$. 
Para modelagem mecânica por elementos finitos é adotado um elemento de placa plana retangular contendo oito nós da família Serendipity (Reddy, 1997) o qual não possui nó central. Desta forma, devido a utilização da teoria FSDT sabe-se que a mesma adota cinco variáveis mecânicas na definição do seu campo de deslocamentos, que podem ser expressas em termos das suas 40 correspondentes variáveis mecânicas nodais $\left\{u_{e}\right\}=\left\{u_{i}, v_{i}, w_{i}, \psi_{x i}, \psi_{y i}\right\}$, com $i$ variando de 1 a 8 , ou seja, se tem cindo graus de liberdade por nó, totalizando 40 graus de liberdade por elemento finito.

A correspondência entre os deslocamentos totais do elemento com a contribuição dos deslocamentos de cada nó é feita por meio das funções de forma, que representam as especificidades de cada tipo de elemento, sendo que, as mesmas já estão representadas em termos das coordenadas locais e definidas em (Faria, 2006).

$$
\{\hat{\mathrm{u}}(\xi, \eta, t)\}=\left[N_{u}(\xi, \eta)\right]\left\{u_{e}(t)\right.
$$

O vetor dos deslocamentos mecânicos e as deformações mecânicas podem ser expressos em termos das funções de forma e das variáveis mecânicas nodais, respectivamente, como segue:

$$
\{U(\xi, \eta, z, t)\}=[A u(z)]\left[N_{u}(\xi, \eta)\right]\left\{u_{e}(t)\right\} ;\{\varepsilon(\xi, \eta, z, t)\}=[D(z)]\left[N_{u}(\xi, \eta)\right]\left\{u_{e}(t)\right\}
$$

Agora, para que seja possível encontrar as matrizes de massa e rigidez elementares, são necessárias algumas integrações. Sabe-se que energia cinética a nível elementar é dada por:

$$
E_{c}=\frac{1}{2} \int_{V_{e}} \rho\{\dot{U}\}^{T}\{\dot{U}\} d V_{e}
$$

onde $\rho$ é a densidade do material, $V_{e}$ é definido como o volume do elemento finito e $\{\dot{U}\}$ é a derivada do vetor dos campos de deslocamentos $\{U\}$, definido na Eq. (1).

Assim, partindo da equação da energia cinética a nível elementar, encontra-se a matriz de massa elementar $\left[M_{e}\right]$, que representa a seguinte integração:

$$
\left[M_{e}\right]=\int_{V_{e}} \rho\left[N_{u}\right]^{T}\left[A_{u}\right]^{T}\left[A_{u}\right]\left[N_{u}\right] d V_{e}
$$

Já a matriz de rigidez elementar é obtida a partir da energia potencial de deformação mecânica:

$$
P_{e}=\iiint_{z \xi \eta}\{\sigma\}^{T}\{\varepsilon\} d \eta d \xi d z
$$

Combinando-se a relação entre os campos de tensão e deformação para o material composto, $\{\sigma\}=\left[C_{t}\right]\{\varepsilon\}$ com a Eq. (7), pode-se chegar à seguinte forma para a matriz de rigidez elementar:

$$
\left[K_{e}\right]=\frac{1}{2} \int_{V_{e}}\left[B_{u}\right]^{T}\left[C_{t}\right]\left[B_{u}\right] d V_{e}
$$

Para a montagem das matrizes de massa e rigidez globais utiliza-se de técnicas clássicas de montagem de elementos finitos, conhecendo-se a conectividade do sistema.

De posse das matrizes globais de massa e rigidez, pode-se obter a seguinte equação do movimento do sistema na forma matricial:

$$
\left[M_{g}\right]\left\{\ddot{u}_{g}\right\}+\left[K_{g}\right]\left\{u_{g}\right\}=\left\{F_{g}\right\}
$$

onde, $M_{g}=\bigcup_{e=1}^{n e l} M_{e}^{(e)}$ e $K_{g}=\bigcup_{e=1}^{n e l} K_{e}^{(e)}$ são as matrizes globais de massa e rigidez, $u_{g}$ é o vetor dos graus de liberdade globais e $F_{g}$ representa o vetor dos esforços generalizados.

\section{MODELAGEM ELETROMECÂNICA}

Os campos de deslocamentos mecânicos são modelados via utilização da camada equivalente única, e os potenciais elétricos são distribuídos de forma discreta ao longo das camadas piezelétricas. Neste contexto, os campos de deslocamentos mecânicos são aproximados via FSDT e o potencial elétrico aproximado da seguinte forma: 


$$
\phi(x, y, z, t)=\sum_{j=1}^{n \operatorname{camadas}+1} L_{j}(z) \phi_{j}(x, y, t)
$$

onde, $\phi_{j}$ é o potencial elétrico de cada interface das camadas ao longo da espessura do composto, sendo o subscrito $j$ ligado às interfaces e $L_{j}$ são as funções de interpolação Lagrangeanas.

A subdivisão do laminado em camadas discretas é que garante a discretização das variáveis elétricas por camadas, sendo o potencial elétrico assumido contínuo em cada camada discreta, e assim tendo uma variação do tipo $\mathrm{C}^{0}$ ao longo da espessura. Os campos de deslocamentos elétricos são obtidos pelo negativo do gradiente do potencial elétrico:

$$
E_{i}=-\nabla \phi_{j}
$$

Sabe-se que estruturas cristalinas da classe $\mathrm{mm} 2$ cobrem os materiais piezelétricos mais frequentemente associados às estruturas inteligentes. A matriz elasto-piezo-dielétrica acoplada do material que corresponde à classe mm2 é expressa na forma matricial de forma completa no trabalho de Faria (2006) e, de forma simplificada com o uso do subscrito $l$ para indicar que não se encontra rotacionada como segue:

$$
\left\{\begin{array}{c}
\sigma_{l} \\
D_{l}
\end{array}\right\}=\left[\begin{array}{cc}
c_{l} & -e_{l}^{T} \\
e_{l} & \chi_{l}
\end{array}\right]\left\{\begin{array}{l}
\varepsilon_{l} \\
E_{l}
\end{array}\right\}
$$

onde, $\sigma_{l}$ é o tensor de tensão mecânica $\left[\mathrm{N} / \mathrm{m}^{2}\right] ; D_{l}$ é o tensor deslocamento elétrico $\left[\mathrm{C} / \mathrm{m}^{2}\right] ; \chi_{l}$ é a matriz de permissividade dielétrica $[\mathrm{N} / \mathrm{Vm}] ; \varepsilon_{l}$ é o tensor de deformação mecânica $[\mathrm{m} / \mathrm{m}] ; E_{l}$ é o tensor campo elétrico $[\mathrm{V} / \mathrm{m}]$ ou $\left[\mathrm{N} / \mathrm{m}^{2}\right] ; c_{l}$ é o tensor de elasticidade linear $\left[\mathrm{N} / \mathrm{m}^{2}\right]$ e $e_{l}$ é o tensor de constantes dielétricas para deformação mecânica constante $\left[\mathrm{N} / \mathrm{V}^{2}\right]$, todos nesse caso dados em coordenadas locais.

Para que tais equações possam ser escritas levando-se em consideração a direção das fibras do composto é necessário fazer a rotação do sistema de coordenadas. Neste caso, serão considerados compostos onde as camadas podem ser rotacionadas em relação ao eixo z. São utilizadas as matrizes $T$ e $Q$ de transformação, visto que, a primeira apropriada para propriedades mecânicas e a segunda para propriedades elétricas. As mesmas encontram-se definidas no trabalho de Faria (2006).

Desta forma, ao se levar em conta a direção das fibras do composto, chega-se á seguinte equação matricial a qual já se encontra rotacionada:

$$
\left\{\begin{array}{c}
\sigma_{g} \\
D_{g}
\end{array}\right\}=\left[\begin{array}{cc}
T c_{l} T^{T} & -T e_{l}^{T} Q^{-1} \\
Q e_{l} T^{T} & Q \chi_{l} Q^{-1}
\end{array}\right]\left\{\begin{array}{c}
\varepsilon_{g} \\
E_{g}
\end{array}\right\}
$$

Obtendo-se então duas expressões, uma para a tensão mecânica e outra para deslocamento elétrico:

$$
\begin{gathered}
\sigma_{g}=c_{g} \varepsilon_{g}-e_{g}^{T} E_{g} \\
D_{g}=e_{g} \varepsilon_{g}+\chi_{g} E_{g}
\end{gathered}
$$

Dada uma estrutura laminada dividida em várias camadas, cada uma das $i$ camadas tem uma função potencial elétrico $\phi_{\text {camada (i) }}$ composta por duas funções de interface $\phi_{i}$ e $\phi_{(i+1)}$ nas interfaces inferior e superior desta camada. Assim o potencial elétrico da $i$-ésima camada é obtido como expresso na Eq. (16):

$$
\phi_{\text {camada }(i)}(x, y, z, t)=L_{i d}(z) \phi_{i}(x, y, t)+L_{i u}(z) \phi_{i+1}(x, y, t)
$$

onde $L_{i d}(z)=\frac{z-z_{i+1}}{z_{i}-z_{i+1}}$ e $L_{i u}(z)=\frac{z-z_{i}}{z_{i+1}-z_{i}}$ são funções de interpolação Lagrangeana Linear da interface inferior e superior, respectivamente, da $i$-ésima camada do composto laminado.

A partir da definição do campo elétrico como sendo o negativo do gradiente do potencial elétrico, e da Eq. (16) para a $i$-ésima camada, tem-se:

$$
\left\{\begin{array}{l}
E_{x}(x, y, z, t) \\
E_{y}(x, y, z, t) \\
E_{z}(x, y, z, t)
\end{array}\right\}=-\left\{\begin{array}{c}
L_{i d}(z) \frac{\partial \phi_{i}(x, y, t)}{\partial x}+L_{i u}(z) \frac{\partial \phi_{i+1}(x, y, t)}{\partial x} \\
L_{i d}(z) \frac{\partial \phi_{i}(x, y, t)}{\partial y}+L_{i u}(z) \frac{\partial \phi_{i+1}(x, y, t)}{\partial y} \\
\frac{1}{z_{i}-z_{i+1}} \phi_{i}(x, y, t)+\frac{1}{z_{i+1}-z_{i}} \phi_{i+1}(x, y, t)
\end{array}\right\}
$$

As funções de interface $\phi_{i}(x, y, t)$ e $\phi_{i+1}(x, y, t)$ para um elemento de placa de 8 nós da família Serendipity podem ser escritas em termos das funções de forma elétrica $N_{\phi}$ (Faria, 2006), as quais se encontram em coordenadas locais. Na 
representação dos potenciais elétricos nodais $\varphi_{i j}$, o subscrito $i$ indica o número da interface da camada, e o subscrito $j$ indica o número local do nó. Para um composto formado por $i$ interfaces:

$$
\{\phi(\xi, \eta, t)\}_{(i+1) x 1}=\left[N_{\phi}(\xi, \eta)\right]_{(i+1) x 8(n+1)}\left\{\varphi_{e}(t)\right\}_{8(i+1) x 1}
$$

Como foi observado, para o caso em que o potencial elétrico é escrito para todas as camadas do composto, a matriz resultante será esparsa, com muitos termos nulos. Neste sentido, é mais interessante escrever o potencial elétrico separadamente para cada uma das camadas. Assim, o potencial elétrico para a $i$-ésima camada é expresso sob a forma:

$$
\phi(\xi, \eta, t)_{i}=\left[N_{\phi}(\xi, \eta, z)\right]_{1 \times 8(n+1)}\left\{\varphi_{e}(t)\right\}_{8(n+1) \times 1}
$$

Desta forma a matriz $N_{\phi}$ relaciona os potenciais elétricos nodais no volume do elemento com os valores do potencial elétrico total de cada camada.

Pode-se então relacionar a matriz das funções de forma elétrica $N_{\phi}$ com o vetor campo elétrico, dado na Eq. (17), da seguinte forma simplificada:

$$
\{E(x, y, z, t)\}_{3 \times 1}=-\left[B_{\phi}(\xi, \eta, z)\right]_{3 \times 8(n+1)}\left\{\varphi_{e}(t)\right\}_{8(n+1) \times 1}
$$

Agora é então possível encontrar as matrizes de massa e rigidez elementares do sistema acoplado. A energia cinética a nível elementar é dada pela Eq. (5), obtendo-se a matriz de massa elementar, da mesma maneira que foi demonstrada na seção anterior, utilizando-se da aproximação dos deslocamentos mecânicos pela teoria da camada equivalente única. Já a obtenção das matrizes de rigidez elementares do elemento finito contendo elemento piezelétrico se dá de uma maneira mais abrangente, visto que, neste caso, os potenciais elétricos são relevantes, podendo os mesmos interferir na rigidez do sistema, sendo modelados pela teoria discreta. Partindo-se da expressão da energia de deformação (Chee, 2000):

$$
U=\int_{V_{e}}\left(\varepsilon^{T} \sigma-E^{T} D\right) d V_{e}
$$

A Equação (21) combinada com as Eqs. (14) e (15), resulta:

$$
U=\int_{V_{e}}\left(\varepsilon_{g}^{T} c_{g} \varepsilon_{g}-\varepsilon_{g}^{T} e_{g}^{T} E_{g}-E_{g}^{T} e_{g} \varepsilon_{g}-E_{g}^{T} \chi_{g} E_{g}\right) d V_{e}
$$

A partir das relações $\varepsilon_{g}(x, y, z, t)=B_{u}(x, y, z) u_{e}(t), E_{g}(x, y, z, t)=B_{\phi}(x, y, z) \varphi_{e}(t)$, pode-se chegar às seguintes matrizes de rigidez do elemento contendo material piezelétrico em coordenadas locais, uma vez que, para se realizar a integração, transforma-se as coordenadas globais $(x, y)$ em coordenadas locais $(\xi, \eta)$, utilizando-se da correlação entre coordenadas e do jacobiano, os quais encontram-se definidos em Faria (2006).

$$
\begin{aligned}
K_{u u}^{e} & =\int_{\xi=-1}^{\xi=1} \int_{\eta=-1}^{\eta=1} \sum_{i=1}^{n} \int_{z_{i}}^{z_{i}+1}\left(B_{u}^{T} c_{g} B_{u}\right) d z J d \eta d \xi \\
K_{u \phi}^{e} & =\int_{\xi=-1}^{\xi=1} \int_{\eta=-1}^{\eta=1} \sum_{i=1}^{n} \int_{z_{i}}^{z_{i}+1}\left(B_{u}^{T} e_{g} B_{\phi}\right) d z J d \eta d \xi \\
K_{\phi u}^{e} & =\int_{\xi=-1}^{\xi=1} \int_{\eta=-1}^{\eta=1} \sum_{i=1}^{n} \int_{z_{i}}^{z_{i}+1}\left(B_{\phi}^{T} e_{g} B_{u}\right) d z J d \eta d \xi \\
K_{\phi \phi}^{e} & =\int_{\xi=-1}^{\xi=1} \int_{\eta=-1}^{\eta=1} \sum_{i=1}^{n} \int_{z_{i}}^{z_{i}+1}\left(B_{\phi}^{T} \chi_{g} B_{\phi}\right) d z J d \eta d \xi
\end{aligned}
$$

Desta forma, a partir das matrizes elementares pode-se obter a seguinte equação do movimento:

$$
\left[\begin{array}{cc}
M_{g} & 0 \\
0 & 0
\end{array}\right]\left\{\begin{array}{l}
\ddot{u} \\
\ddot{\varphi}
\end{array}\right\}+\left[\begin{array}{ll}
K_{u u} & K_{u \phi} \\
K_{\phi u} & K_{\phi \phi}
\end{array}\right]\left\{\begin{array}{l}
u \\
\varphi
\end{array}\right\}=\left\{\begin{array}{l}
F \\
Q
\end{array}\right\}
$$

onde, $M_{g}=\sum_{e=1}^{n e} L_{e}^{T} M^{e} L_{e}$ sendo $M^{e}=\sum_{i=1}^{n} M_{i}^{e} ; K_{u u}=\sum_{e=1}^{n e} L_{e}^{T} K_{u u}^{e} L_{e} \quad ; \quad K_{u \phi}=\sum_{e=1}^{n e} L_{e}^{T} K_{u \phi}^{e} L_{e} \quad ; \quad K_{\phi \phi}=$ $\sum_{e=1}^{n e} L_{e}^{T} K_{\phi \phi}^{e} L_{e}$, sendo a matriz $L_{e}$ a matriz de correspondência entre os graus de liberdade elementares e os globais.

\section{INCLUSÃO DOS CIRCUITOS SHUNT}

Nesta parte do trabalho ênfase é dada à introdução dos circuitos elétricos shunt no sistema de equações do movimento acopladas. Neste sentido, a Eq (24) pode ser reescrita em duas expressões:

$$
\left[M_{g}\right]\{\ddot{u}(t)\}+\left[K_{u u}\right]\{u(t)\}+\left[K_{u \phi}\right]\{\phi(t)\}=\{f(t)\}
$$




$$
\left[K_{\phi u}\right]\{u(t)\}+\left[K_{\phi \phi}\right]\{\phi(t)\}=\{q(t)\}
$$

No sistema piezo-shunt são considerados dois eletrodos na pastilha piezelétrica. Os nós que constituem cada eletrodo são equipotenciais elétricos. Esta condição é considerada introduzindo uma transformação no vetor dos potenciais elétricos. Além disso, para a análise no domínio da frequência, a Eq. (24) pode ser representada no domínio de Fourier, negligenciando condições iniciais, levando às equações do movimento:

$$
\begin{gathered}
\left(K_{u u}-\omega^{2} M_{g}\right) U(\omega)+\bar{K}_{u \phi} \bar{\phi}(\omega)=F(\omega) \\
\bar{K}_{\phi u} U(\omega)+\bar{K}_{\phi \phi} \bar{\phi}(\omega)=\bar{Q}(\omega)
\end{gathered}
$$

onde $\bar{\phi}(\omega)$ designa o vetor formado pelos potenciais elétricos que permanecem independentes entre si.

As equações do movimento devem ser transformadas para considerar o tipo de circuito shunt conectado aos eletrodos, admitindo-se a transferência das cargas elétricas entre os mesmos.

O vetor de correntes elétricas que fluem através dos circuitos shunt, cujas impedâncias formam a matriz, $Z(\omega)$, é:

$$
\bar{Q}(\omega)=(1 / j \omega) Z^{-1}(\omega) L \bar{\phi}(\omega)
$$

Na Equação (27), a matriz $L$ permite selecionar, dentre os potenciais elétricos independentes, aqueles que correspondem aos eletrodos dos circuitos shunt conectados. Combinando-se as equações (26-b) e (27), obtém-se:

$$
\bar{K}_{\phi u} U(\omega)+\left(\bar{K}_{\phi \phi}-\frac{Z^{-1}(\omega)}{j \omega} L\right) \bar{\phi}(\omega)=0
$$

As Equações (26-a) e (28) podem ser combinadas em termos dos graus de liberdade mecânicos, exclusivamente, permitindo obter a seguinte matriz de Funções de resposta em frequência (FRFs):

$$
H(\omega)=\left[\bar{K}_{u u}-\bar{K}_{u \phi}\left(\bar{K}_{\phi \phi}-\frac{1}{j \omega} Z^{-1}(\omega)\right)^{-1} \bar{K}_{\phi u}-\omega^{2} M_{g}\right]^{-1}
$$

A equação acima pode ser utilizada para diferentes tipos de circuitos shunt mediante a introdução das expressões correspondentes de suas impedâncias elétricas, indicadas por $Z(\omega)$, sendo as mesmas diferentes para cada tipo de circuito. Para o caso do circuito shunt resistivo, $\mathrm{Z}=R$, já para o caso do circuito shunt ressonante, $Z=R+j X(w)$. Desta forma, o circuito resistivo por apresentar apenas resistência, é composto apenas de parte real, enquanto que o ressonante apresenta além da parte resistiva, a parte indutiva, sendo composto então tanto por componente real quanto imaginária.

\section{SIMULAÇÕES NUMÉRICAS}

Nesta seção, foram realizadas simulações numéricas utilizando-se uma placa composta em balanço, formada por cinco camadas, das quais, quatro delas são de material composto e, a última, de um elemento piezelétrico. A placa foi discretizada em 6 elementos finitos, como ilustrado na Fig. 1. Além disso, foi investigada a seguinte configuração para o composto: $0^{\circ} / 90^{\circ} / 90^{\circ} / 0^{\circ}$. As simulações em questão foram comparadas com Zambolini-Vicente (2014).

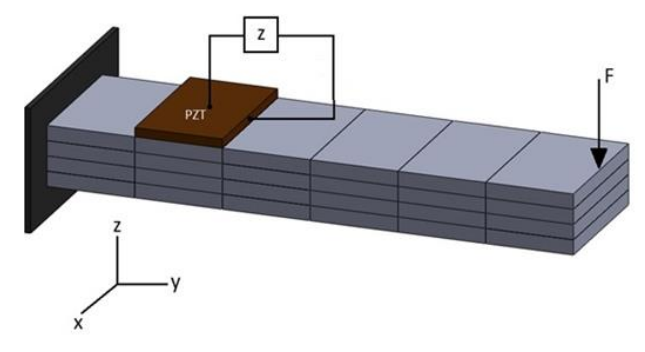

Figura 1 - Placa composta em balanço com elemento piezelétrico

O problema apresentado na Fig. 1 é constituído de uma viga de material composto com densidade $\rho=$ $1578 \mathrm{Kg} / \mathrm{m}^{3}$, em balanço, formada por quatro camadas contendo um elemento piezelétrico (PZT) de densidade $\rho=7600 \mathrm{Kg} / \mathrm{m}^{3}$. Neste elemento serão acoplados circuitos elétricos shunt do tipo resistivo e ressonante. Em seguida os resultados serão comparados para avaliar o desempenho dos mesmos. Na mesma figura, está apresentada a discretização por elementos finitos do sistema a ser estudado, sendo que, a espessura de cada camada do composto é de 
$1,048.10^{-4} \mathrm{~m}$, a largura, tanto da viga quanto do PZT, dada por $0,0254 \mathrm{~m}$, e o comprimento total da viga de 0,3048 m. A espessura do PZT utilizado é de 0,762 $\mathrm{mm}$, comprimento de 0,0508 $\mathrm{m}$ e a distância entre o mesmo e o engaste sendo de 0,0020 m. Nas Tabelas 1 e 2 são apresentadas, respectivamente, as propriedades mecânicas e elétricas dos materiais envolvidos para a geração do modelo de elementos finitos do sistema eletromecânico.

Tabela 1 - Propriedades mecânicas do composto e do PZT G1195

\begin{tabular}{lccccccccc}
\hline Material & $C_{11}$ & $C_{12}$ & $C_{13}$ & $C_{22}$ & $C_{23}$ & $C_{33}$ & $C_{44}$ & $C_{55}$ & $C_{66}$ \\
\hline Composto & $1,72 \times 10^{1}$ & $1,48 \times 10^{9}$ & $1,48 \times 10^{9}$ & $7,58 \times 10^{9}$ & $2,28 \times 10^{9}$ & $7,58 \times 10^{9}$ & $1,38 \times 10^{9}$ & $3,45 \times 10^{9}$ & $3,45 \times 10^{9}$ \\
PZT & $1,02 \times 10^{1}$ & $5,03 \times 10^{14}$ & $5,03 \times 10^{14}$ & $1,02 \times 10^{15}$ & $5,03 \times 10^{14}$ & $1,02 \times 10^{15}$ & $2,59 \times 10^{14}$ & $2,59 \times 10^{14}$ & $2,59 \times 10^{14}$ \\
\hline
\end{tabular}

Tabela 2 - Propriedades eletromecânicas e elétricas do PZT G1195

\begin{tabular}{lcccccccc}
\hline Material & $\begin{array}{c}e_{15} \\
\left(C / m^{2}\right)\end{array}$ & $\begin{array}{c}e_{24} \\
\left(C / m^{2}\right)\end{array}$ & $\begin{array}{c}e_{31} \\
\left(C / m^{2}\right)\end{array}$ & $\begin{array}{c}e_{32} \\
\left(C / m^{2}\right)\end{array}$ & $\begin{array}{c}e_{33} \\
\left(C / m^{2}\right)\end{array}$ & $\begin{array}{c}\chi_{11} \\
(F / m)\end{array}$ & $\begin{array}{c}\chi_{22} \\
(F / m)\end{array}$ & $\begin{array}{c}\chi_{33} \\
(F / m)\end{array}$ \\
\hline PZT & 0 & 0 & $-18,2998$ & $-9,0133$ & $-9,0133$ & $1,593 \times 10^{-8}$ & $1,593 \times 10^{-8}$ & $1,593 \times 10^{-8}$ \\
\hline
\end{tabular}

Os valores nominais de resistência, indutância e espessura do piezelétrico foram calculados de acordo com a literatura como sendo ótimos, os quais são mostrados na Tab. 3, (Hagood e von Flotow, 1991).

Tabela 3 - Valores nominais de Resistência, Indutância e Espessura

\begin{tabular}{cccc}
\hline Tipo circuito & Resistência $[\Omega]$ & Indutância $[H]$ & Espessura $[\mathrm{m}]$ \\
\hline Shunt resistivo & $3,23 \times 10^{5}$ & 0 & $7,62 \times 10^{-4}$ \\
Shunt ressonante & $1,61 \times 10^{4}$ & $6,29 \times 10^{2}$ & $7,62 \times 10^{-4}$ \\
\hline
\end{tabular}

Assim, observa-se um alto valor de indutância para o caso do circuito ressonante. A primeira simulação compara as FRFs da estrutura sem nenhum tipo de circuito e, a mesma com shunts resistivo e ressonante acoplados, sendo que, a Fig. 2(a), compara a estrutura sem nenhum tipo de circuito e a mesma com shunt resistivo acoplado e a Fig. 2(b) compara a estrutura sem circuito e a mesma com circuito ressonante acoplado.
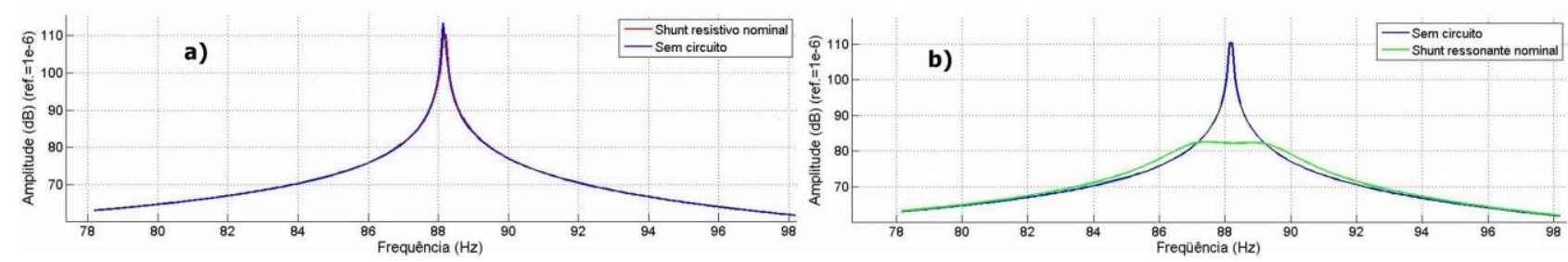

Figura 2 - Comparação das FRFs para o sistema sem e com circuito shunt: a) resistivo; b) ressonante

Da Figura 2, pode-se notar que praticamente não ocorreu deslocamento da frequência de ressonância ao se acoplar os circuitos shunts à estrutura. Entretanto, fica claro a superioridade do circuito shunt ressonante, visto que, houve uma redução significativa de cerca de $25[d B]$ na amplitude da resposta em frequência para o modo em questão.

\section{OTIMIZAÇÃO MULTIOBJETIVO DETERMINÍSTICA}

Para que fosse possível encontrar os valores ótimos de resistência, indutância e espessura do elemento piezelétrico utilizou-se o algoritmo genético NSGA (Non-dominated Sorting Genetic Algorithm), baseado na construção de frentes de Pareto formadas por conjuntos de soluções ótimas no espaço multiobjetivo de projeto. As funções objetivo, como podem ser observadas nas figuras abaixo, foram $f 1$ que corresponde à amplitude do $1^{\circ}$ modo de vibração da estrutura e, $f 2$ que corresponde à massa total acrescida à estrutura composta pelo elemento piezelétrico. Foram escolhidos os pontos que melhor satisfazem o compromisso dessas duas funções objetivo, sendo que, os mesmos encontram-se circulados na Fig. 3 que mostra as frentes de Pareto obtidas com a otimização.

Tabela 4 - Parâmetros utilizados no algoritmo NSGA

\begin{tabular}{cccccc}
\hline $\begin{array}{c}\text { Probabilidade de } \\
\text { seleção }\end{array}$ & $\begin{array}{c}\text { Probabilidade de } \\
\text { reprodução }\end{array}$ & $\begin{array}{c}\text { Probabilidade de } \\
\text { mutação }\end{array}$ & $\begin{array}{c}\text { Número de } \\
\text { gerações }\end{array}$ & $\begin{array}{c}\text { Número de } \\
\text { indivíduos }\end{array}$ & $\begin{array}{c}\text { Coeficiente de } \\
\text { niche }(\sigma)\end{array}$ \\
\hline 0,25 & 0,25 & 0,25 & 50 & 50 & 0,2 \\
\hline
\end{tabular}



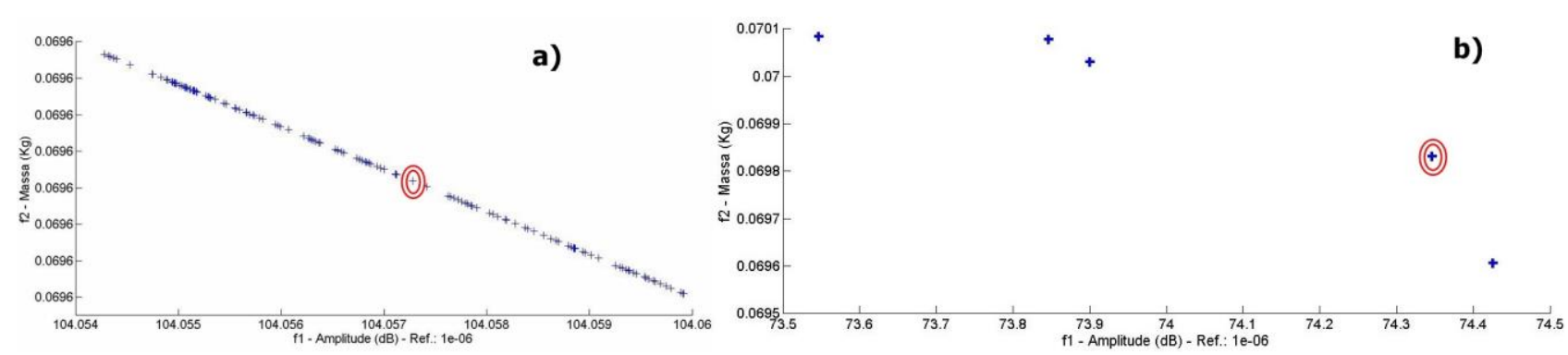

Figura 3 - Frentes de Pareto. a) Shunt resistivo; b) Shunt ressonante

Os parâmetros ótimos que melhor satisfazem as relações entre as funções objetivo, tanto para o shunt resistivo otimizado, quanto para o ressoante, são listados na Tab. 5. É importante destacar que a escolha dos parâmetros ótimos referente a um ponto no espaço de Pareto, é feita levando-se em conta o melhor compromisso das soluções.

Tabela 5 - Valores ótimos de Resistência, Indutância e Espessura

\begin{tabular}{cccc}
\hline Tipo circuito & Resistência $[\Omega]$ & Indutância $[H]$ & Espessura $[\mathrm{m}]$ \\
\hline Shunt resistivo & $28,99 \times 10^{3}$ & 0 & $3,05 \times 10^{-4}$ \\
Shunt ressonante & $18,86 \times 10^{3}$ & $3,05 \times 10^{2}$ & $3,10 \times 10^{-4}$ \\
\hline
\end{tabular}

De posse dos parâmetros ótimos para cada tipo de circuito, foram calculadas as respectivas funções de resposta em frequência como mostrado na Fig. 4. Observa-se o elevado valor de indutância calculado para o caso do circuito shunt ressonante, levando-se assim, a necessidade de utilização de indutâncias sintéticas para o caso experimental.
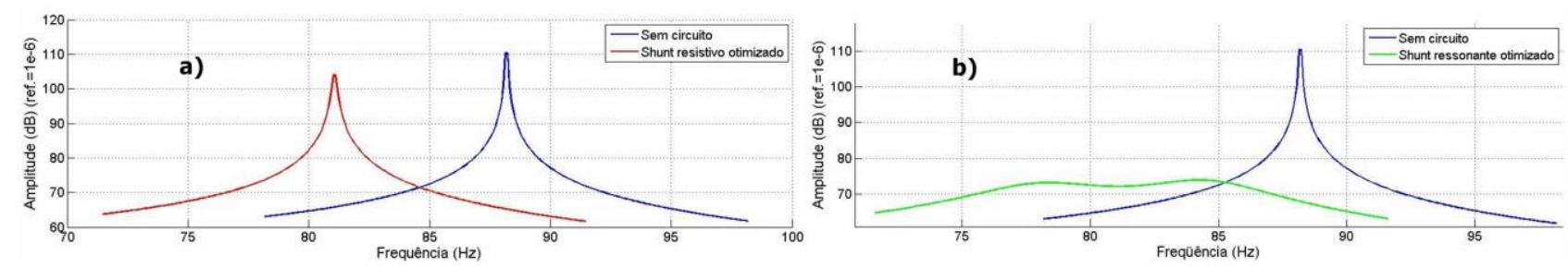

Figura 4 - Comparação das FRFs para o sistema sem e com shunt otimizado: a) resistivo; b) ressonante

Assim, nota-se claramente uma maior eficiência do circuito otimizado em termos da redução das amplitudes das vibrações. Entretanto, observa-se também que houve uma alteração significativa no sistema em função da diminuição da frequência de ressonância, a qual estava compreendida na banda presente entre $80[\mathrm{~Hz}]$ e $95[\mathrm{~Hz}]$ e que passou para a banda de $70[\mathrm{~Hz}]$ e $90[\mathrm{~Hz}]$. Neste exemplo fica evidente a importância da otimização dos parâmetros do circuito para que se tenha melhores resultados na redução de amplitudes de vibrações.

Para o caso da estrutura composta contendo circuito shunt ressonante otimizado foi possível observar uma queda brusca na amplitude da função de resposta em frequência, passando de $110[\mathrm{~dB}]$ na banda de frequência compreendida entre $80[\mathrm{~Hz}]$ e $95[\mathrm{~Hz}]$ para cerca de $75[\mathrm{~dB}]$ em uma nova banda $70[\mathrm{~Hz}]$ e $90[\mathrm{~Hz}]$, atingindo assim com êxito o objetivo do trabalho, que é a redução das amplitudes de vibração da estrutura além de demonstrar a superioridade do circuito ressonante em relação ao resistivo. Observa-se também nessa figura a geração de dois picos de antirressonância após o acoplamento do circuito shunt ressonante a estrutura.

\section{CONCLUSÕES GERAIS}

Neste trabalho foi apresentado o projeto ótimo determinístico e a modelagem por elementos finitos de placas planas compostas contendo elementos piezelétricos acoplados a circuitos elétricos shunt para o controle passivo de vibrações. Diante dos resultados obtidos, pode-se concluir que:

a) Comparando as amplitudes das FRFs da estrutura composta com shunt resistivo e ressonante otimizados, podese notar que para ambos os casos, houve alterações significativas na frequência de ressonância do sistema. Isto pode ser explicado em função da alteração nos valores geométricos da estrutura como a espessura do elemento piezelétrico. Além disso, é possível observar que o efeito do circuito shunt resistivo é análogo ao de se atribuir características viscoelásticas ao sistema, enquanto que o comportamento induzido pelo circuito shunt resistivoindutivo é similar ao observado com a inclusão de um absorvedor dinâmico de vibrações.

b) O circuito ressonante mostra ser mais vantajoso em termos da redução dos níveis de vibrações do sistema quando comparado com o circuito elétrico resistivo. 
c) Como as frequências envolvidas em problemas de controle passivo de vibrações são baixas, como demonstrado nas simulações numéricas, as indutâncias requeridas no caso do uso de circuito shunt ressonante para o controle passivo de vibrações são muito elevadas. Tal fato leva a necessidade de utilização de indutâncias sintéticas.

d) Em termos do uso de técnicas de otimização determinística, os resultados obtidos permitem concluir sobre a importância de se considerar não somente os parâmetros do circuito elétrico, mas também da estrutura, mesmo tendo sido considerado aqui somente a espessura do elemento piezelétrico.

\section{AGRADECIMENTOS}

À Faculdade de Engenharia Mecânica da Universidade Federal de Uberlândia e ao Instituto Nacional de Ciência e Tecnologia de Estruturas Inteligentes em Engenharia (INCT-EIE) pela oportunidade de realizar este trabalho.

À CAPES (Coordenação de Aperfeiçoamento de Pessoal de Nível Superior) pela bolsa de estudos concedida para realização do trabalho e aos órgãos de fomento CNPq (Conselho Nacional de Desenvolvimento Científico e Tecnológico) e FAPEMIG (Fundação de Amparo à Pesquisa do Estado de Minas Gerais) pelo suporte financeiro.

\section{REFERÊNCIAS}

Berthelotb, J. M., 1992. Matériaux Composites: Comportement Mécanique et Analyse des Structures. Paris: Masson.

Callister, Jr., W.D., 2002. Ciência e engenharia de materais: uma introdução. Editora LTC, 5 Ed., Rio de Janeiro, RJ. ISBN: 85216-1288-5

Cen, S., Soh, A., Long, Y.; Yao, Z. A., 2002. New 4-node Quadrilateral FE Model with Variable Electrical Degrees of Freedom for the Analysis of Piezoelectric Laminated Composite Plates. Composite Structures. v. 58, n.4, p. 583599.

Chee, C. Y. K., 2000. Static Shape Control of Laminated Composite Plate Smart Structure using Piezoelectric Actuators. Tese de Pós-Doutorado.- University of Sydney: Department of Aeronautical Engineering, Sydney, Austrália.

Chugal, Y. M.; Shimpi, R. P., 2002. A Review of Refined Shear Deformation Theories of Isotropic and Anisotropic Laminated Plates. Journal of Reinforced Plastics and Composites. v. 21, n. 9.

Clark, R. L., Saunders, W. R. e Gibbs, G. P., 1998, "Adaptive Structures - Dynamics \& Control", John Wiley \& Sons, Inc.

Faria, A. W. ; Lima, A. M. G. ; Rade, R. S. L. ; Rade, D. A., 2008. Avaliação numérica por elementos finitos do amortecimento em materiais compostos laminados. In: V Congresso Nacional de Engenharia Mecânica, Salvador. Anais do V Congresso Nacional de Engenharia Mecânica.

Faria, A. W., 2006. Modelagem por elementos finitos de placas compostas dotadas de sensores e atuadores piezoelétricos: implementação computacional e avaliação numérica. 152f. Dissertação de Mestrado, Universidade Federal de Uberlândia, Uberlândia, MG.

Hagood, N. W. e von Flotow, A., 1991, "Damping of Structural Vibrations with Piezoelectric Materials and Passive Electrical Networks", Journal of Sound and Vibration, Vol. 146, №2, pp 243-268.

Mendonça, P. T. R., 2005. Materiais Compostos \& Estruturas - Sanduíche: Projeto e Análise 1.ed. Manole.

Pinheiro, M. A. S. ; Beckhauser, G. N. ; Menezes, M. R., 2006. Aplicativo Voltado para a Macromecânica Básica das Estruturas Laminadas de Material Compósito. Boletim SBMAC (Rio de Janeiro), Rio de Janeiro, v. VII, p. $209-222$.

Reddy, J. N., 1997. Mechanics of Laminated Composite Plates: Theory and Analysis. 2.ed. Florida: CRC Press.

Viana, F.A.C., 2005. Amortecimento de vibrações usando pastilhas piezoelétricas e circuitos shunt ressonantes.

Zambolini-Vicente, B. G. G. L., 2014. Dissertação de Mestrado em Engenharia Mecânica, Universidade Federal de Uberlândia, Uberlândia. Projeto robusto de circuitos shunt para o controle passivo de vibrações de estruturas compostas. $97 \mathrm{f}$.

\section{RESPONSABILIDADE AUTORAL}

Os autores são os únicos responsáveis pelo conteúdo deste trabalho. 


\title{
OPTIMAL DESIGN OF SHUNT CIRCUITS FOR THE PASSIVE CONTROL OF VIBRATION OF COMPOSITE STRUCTURES
}

\author{
Lorrane Pereira Ribeiro, lorranemecatronica@gmail.com ${ }^{1}$ \\ Antônio Marcos Gonçalves de Lima, amglima @ mecanica.ufu.br ${ }^{2}$ \\ ${ }^{1,2}$ Federal University of Uberlândia - School of Mechanical Engineering - Av. João Naves de Ávila, 2121, Santa \\ Mônica, 38408-100, Uberlândia, Minas Gerais.
}

\begin{abstract}
This work is devoted to the numerical and computational modeling of composite structural plate likestructures incorporating piezoelectric elements to be coupled with shunted circuits with the aim of passive vibrations attenuation. Also, in the design procedure, deterministic multiobjective optimization strategies will be considering in order to obtain the optimal shunt circuits. In the context of the finite element modeling procedure, emphasis is placed on the combination of the equivalent single layer theory and the so-named First-Order Shear Deformation Theory (FSDT) with Layerwise functions to taking into account the discrete electrical fields for piezoelectric paths. The considered finite element based modeling methodology has been implemented computationally by using the commercial available MATLAB® programming code. Thus, based on the available computational programs it is possible to construct an optimal shunt piezoelectric circuit to be applied on a more complex composite engineering system of industrial interest for vibration abatement. The numerical results obtained for a composite beam likestructure with non-optimal shunt circuits and optimal shunt circuit demonstrate the performance of the shunt circuits to reduce the undesirable vibrations of engineering structures.
\end{abstract}

Palavras-chave: composite structures, piezoelectric materials, shunt circuits, passive control of vibration 